\title{
Extrinsic noise acts to lower protein production at higher translation initiation rates
}

\author{
Rati Sharma ${ }^{1, *}$ \\ ${ }^{1}$ Department of Chemistry, \\ Department of Data Science and Engineering, \\ Indian Institute of Science Education and Research (IISER) Bhopal \\ Bhopal Bypass Road, Bhauri, Bhopal 462066 INDIA
}

\begin{abstract}
Any cellular process at the microscopic level is governed by both extrinsic and intrinsic noise. In this article, we incorporate extrinsic noise in a model of mRNA translation and carry out stochastic simulations of the same. We then evaluate various statistics related to the residence time of the ribosome on the mRNA and subsequent protein production. We also study the effect of slow codons. From our simulations, we show that noise in the translation initiation rate rather than the translation termination rate acts to significantly broaden the distribution of mRNA residence times near the membrane. Further, the presence of slow codons acts to increase the mean residence times. However, this increase also depends on the number and position of the slow codons on the lattice. We also show that the the slow codons act to mask any effect from the extrinsic noise themselves. Our results have implications towards a better understanding of the role the individual components play during the translation process.
\end{abstract}

\section{INTRODUCTION}

Despite having the same genetic information, the phenotypes of isogenic cells are not the same due to a variation in the protein expression among them. This variation is due to a combination of intrinsic and extrinsic fluctuations that influence the expression of proteins [13]. In a biochemical system that consists of multiple reactants and products, the stochasticity in the system increases multiple folds. This results in an inherent randomness known as Intrinsic Noise (IN). The environment in which these biochemical reactions occur, introduces multiple stochastic variables (such as shared resources among populations of cells or genetic circuits and promoter architecture or pathways upstream of the circuit of interest, among others) that contribute to the Extrinsic Noise (EN) [2, 4, 5]. Noisy gene expression is known to be beneficial under conditions that are stressful or are prone to change. This lets a cell hedge its bet and adapt to changing scenarios [6-10]. Study of the effects of noise on gene expression can therefore lead to a better understanding of the phenotypic variability in these systems.

\footnotetext{
*rati@iiserb.ac.in
}

The fluctuations in protein expression can occur at different stages of the central dogma such as transcription, translation and post translational modification $[1,2,11-$ 14]. In this work, we focus on the stochasticity inherent in mRNA translation, which itself is a complex process. The translation process broadly consists of three main stages, viz. initiation, elongation and termination. The ribosome machinery binds to the 5' end of the mRNA during the translation inititation stage. This ribosome assembly then moves from codon to codon on the mRNA, in the process, adding amino acids to the growing oligomeric chain. This is the elongation stage. Finally, ribosomes exit the mRNA at the 3' end, terminating the process of translation [15].

Even though the basic bits and pieces of central dogma have already been constructed, mRNA translation is still an active area of research. Several experimental studies are now looking at how the individual components of translation affect and aid protein synthesis [15-21]. These studies have also inspired several modeling frameworks that look at mRNA translation as a whole [2228 , effects of the assembly of the ribosomal machinery $[25,29]$ or protein expression bursts as a result of stochastic transcription and translation $[2,4,11,30-40]$. In this 
work, we focus on the kinetic aspects of mRNA translation. Specifically, like any cellular process, the three stages of translation are stochastic in nature and in the context of the translation process account for intrinsic noise. However, the rates of the three stages can themselves be stochastically fluctuating, which has its basis in extrinsic noise (EN) [36, 38, 41].

Here, we study the effects of EN on mRNA translation, which in turn has an effect on the ribosome residence time of the mRNA and the number of proteins produced. Further, we also look at the effect of slow codons on these properties. In section II, we describe the TASEP framework used to model mRNA translation. Section III gives a brief background and mathematical framework of extrinsic noise. Sections IV and V discuss the results and conclusions of this study.

\section{TRANSLATION MODEL AND THEORETICAL BACKGROUND}

In bacteria, the process of translation takes place in the cytoplasm with the help of diffusing ribosomes. Further, several experiments exploring the sub-cellular spatial organization of bacterial cells, have also led to the observation that there are certain proteins that bind to the membrane and localize in specific pockets [42-45]. The mRNAs that code for these membrane binding proteins lie very close to the inner membrane of the bacterial cell. These observations lead to the hypothesis of cotranslational insertion [46, 47].

This system of cotranslational insertion has been theoretically studied using the Totally Asymmetric Exclusion Process (TASEP) framework [26]. This model is also one of the many that study ribosome dynamics on the mRNA, making use of the TASEP framework [22, 25, 27, 29]. The framework, in general, models unidirectional stochastic motion of particles on a onedimensional lattice. The particles themselves are assumed to be point particles with the dynamical constraint that they can only hop on to the neighboring lattice site if it is unoccupied. Otherwise, the particles stay put where they are [48-51]. This system is therefore similar to the process of translation where ribosomes move from codon to codon on the mRNA from the 5' end to the 3' end in a unidirectional manner.

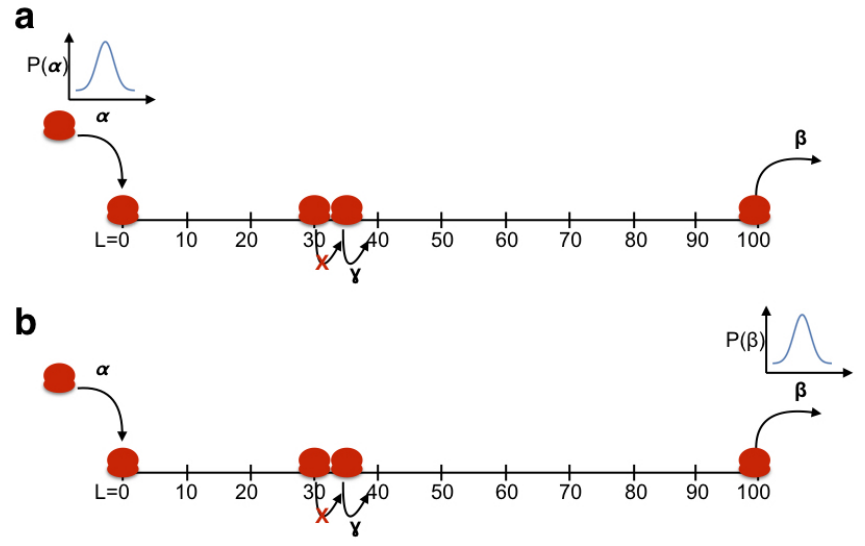

Figure 1. Extrinsic noise in the TASEP model of mRNA translation. Depiction of noise in the rates of (a) translation initiation (rate $\alpha$ ) and (b) translation termination (rate $\beta$ ). The red blobs represent the ribosome assembly. A ribosome enters the mRNA from the 5' end. It then moves from codon to codon (if the site is unoccupied) with rate $\gamma$ in a unidirectional manner towards the 3 ' end, in the process adding amino acids to the growing oligomeric chain. It exits the mRNA once it reaches the 3' end.

It is known that the translation initiation rate depends on the kinetics of the assembly of the ribosome machinery onto the mRNA, specifically on RNA unfolding kinetics and the affinity of a ribosome for the ribosome binding site [52]. In addition to this, the initiation rate is also prone to posttranscriptional regulation [53]. These can make the translation initiation rate dynamically fluctuating.

The translation elongation rates are also generally not uniform across codons on the mRNA [23, 54]. In fact, codons for which the tRNAs are rarely available in the cell tend to have ribosomes stuck on it for much longer than normal. These codons, termed slow codons, cause subsequent slowing down of the protein synthesis not just for that particular ribosome but also for those that come after it [54]. These ribosomal traffic jams therefore lead to longer ribosomal residence times and changes in the gap distribution. As defined in an earlier study [26], we consider the average ratio of the rates $\alpha$ to $\gamma$ to vary between 0.01 to 0.4 . Also, we set the translation elongation rate $(\gamma)$ to be equal to the the translation termination rate $(\beta)$ as they are generally of the same order $[52,53,55]$. 
In our study of extrinsic noise on mRNA translation, we use the TASEP framework [26] to model ribosome dynamics on the mRNA lattice. In this model, a ribosome binds to the mRNA with rate $\alpha$ at the 5' end. The ribosome then moves on to the subsequent codons on the mRNA lattice with rate $\gamma$. Once the ribosome reaches the 3 ' end, it exits the mRNA with rate $\beta$. Our model of the mRNA is assumed to have 100 lattice sites, each site accounting for 10 codons [26] (corresponding to $30 \mathrm{nu}-$ cleotides, which is the average ribosomal footprint of $E$. coli [21]). TASEP models which include full extensions for ribosomal footprint and therefore incorporate exclusion interaction lengths between two ribosomes do not give significantly different results from the simpler model [56]. Therefore, similar to a recent study [26], we proceed to use the simple TASEP framework for our system.

We study the effects of EN on such a translation mechanism by incorporating fluctuating rates of translation initiation and termination. A schematic of this framework is depicted in Fig. 1. We carry out a Monte Carlo simulation study of this system to account for the effects of EN on ribosome translation and protein production. We also look at these effects in the presence of a single or multiple slow codons in the mRNA.

In the next section, we describe the details of incorporating EN into the system.

\section{INCORPORATING EXTRINSIC NOISE INTO MRNA TRANSLATION}

Extrinsic noise can be incorporated into the translation initiation rate $(\alpha)$ or the translation termination rate $(\beta)$, by multiplying the kinetic rate constants with a random number picked from a previously specified distribution. In this work, we study the effect of EN through OrnsteinUhlenbeck (OU) process, a common noise distribution used in several studies [33, 36, 57-59].

Ornstein-Uhlenbeck (OU) process is a stochastic Markov process (represented here by the function $\xi(t)$ ), whose dynamics is specified by the following differential equation.

$$
\frac{\mathrm{d} \xi(t)}{\mathrm{d} t}=-\frac{\xi(t)}{\tau}+c^{1 / 2} \Gamma(t)
$$

Here, $\tau$ is the relaxation time, $\Gamma(t)$ is the Gaussian white noise and $c$ is the diffusion constant given by $c=2 D / \tau$.
$\mathrm{D}$ is a measure of the noise strength, which specifies the deviation of the noise from its mean. The OU process has an exponential time autocorrelation function given by

$$
\left\langle\xi(t) \xi\left(t^{\prime}\right)\right\rangle=D e^{-\left|t-t^{\prime}\right| / \tau}
$$

Thus, $\xi(t)$ and $\xi\left(t^{\prime}\right)$ lose correlation when $\left|t-t^{\prime}\right|$ is greater than $\tau$. Dynamics of $\xi(t)$ is simulated using the following discrete equation [33].

$$
\xi(t+\Delta t)=e^{-\Delta t / \tau} \xi(t)+\left[D\left(1-e^{-2 \Delta t / \tau}\right)\right]^{1 / 2} \eta(t)
$$

where $\eta(t)$ is a unit normal random variable. In our simulations, we start with the initial condition of $\xi(0)=0$, which leads to $\langle\xi\rangle=0$, where the angular brackets denote an average over all realizations of the noise. We also carry out all our simulations with $\Delta t=0.01 \mathrm{~s}$, which is much smaller than or equal to the the value of $\tau$ used to incorporate EN.

In our study, at each time step of the simulation, $\xi(t)$ is picked from the OU process for specific values of $\tau$ and $D$. Subsequently, EN is incorporated into rate constants by replacing $\alpha$ and $\beta$ with $\alpha e^{\xi(t)}$ and $\beta e^{\xi(t)}$ respectively. The intensity of the noise is varied by varying $\tau$ and $D$. A higher $\tau$ corresponds to higher correlation time leading to an increase in EN. Similarly, a high $D$ corresponds to a broader distribution (increased variance) of EN, thereby increasing the chances of higher rates being incorporated into the system.

We carry out Monte Carlo simulations of our TASEP model without extrinsic noise using the well known Gillespie algorithm [60]. The simulations of the model in the presence of extrinsic noise are carried out using the EXTRANDE algorithm, which is a modified version of the Gillespie algorithm, developed to simulate a network in a dynamically fluctuating environment [61].

\section{RESULTS}

\section{A. Ribosome residence times}

As a first step, we study the effect of EN on the residence time distribution of the ribosome along the mRNA lattice. It has been shown that the residence time on a lattice of length $l$ follows a gamma distribution [26], given 

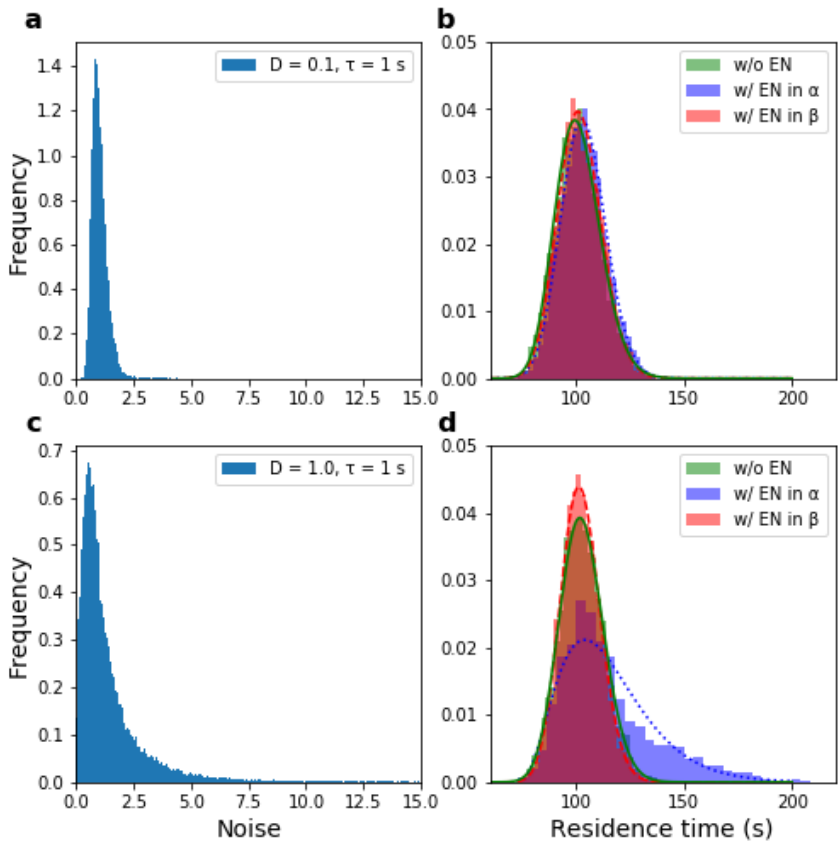

Figure 2. Effect of EN on ribosome residence times.

(a) Simulated OU noise distribution that is incorporated either in the translation initiation rate $(\alpha)$ or the translation termination rate $(\beta)$ with $D=0.1$. (b) Distribution of residence times of ribosomes on the mRNA lattice without EN (green) and when incorporating EN from an OU process in the translation initiation rate, $\alpha$ (blue) and the translation termination rate, $\beta$ (red) for the same values of $D$ as in (a). (c and d) Same as (a and b) but for $D=0.1$. All the other parameters are fixed at $\tau=1.0 \mathrm{~s},\langle\alpha\rangle=0.4 \mathrm{~s}^{-1},\langle\gamma\rangle=1.0 \mathrm{~s}^{-1}$ and $\langle\beta\rangle=1.0 s^{-1}$. Histograms are obtained from simulations and solid lines are fits to gamma distributions. The overlaps in the distributions are shaded darker and in a different colour.

by,

$$
\Gamma(l, t)=\gamma^{l} \frac{t^{l-1}}{(l-1) !} e^{-\gamma t}
$$

where $\gamma$ is the time correlation constant with dimensions $s^{-1}$. In Figs. 2(a and c), we show the EN distributions for two different values of $D$, while keeping $\tau$ fixed. These EN distributions are then incorporated into the kinetic rates of initiation or termination to obtain the ribosome residence time distributions from simulations of the ribosome trafficking. These are shown in Figs. 2(b and d). From the simulations, it is observed that noise in $\alpha$ broadens the residence time distribution compared to the no EN condition, whereas, the distribution for the case when the noise is incorporated in $\beta$ is similar to the no EN condition. One can also observe deviations from the gamma distribution when $\mathrm{EN}$ is incorporated in $\alpha$. Since EN in $\alpha$ shows greater effect on residence times of ribosomes, most of the following analyses are carried out with stochastically fluctuating $\alpha$.

\section{B. Exploring specific effects of the EN parameters}

Protein production from mRNA depends on the amount of time a ribosome takes to traverse the complete mRNA and finally exit it at the 3' end. Through our simulations, we calculate this ribosome residence time, distributions of which are shown in Fig. 2. Production of a single complete protein is accounted for upon the exit of a ribosome from the mRNA.
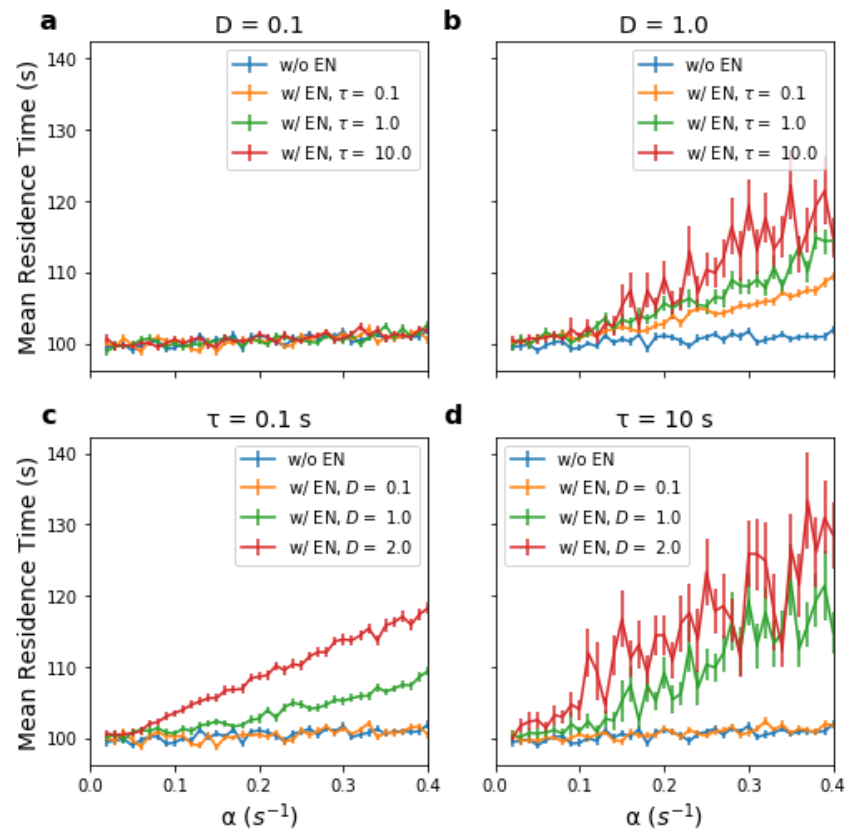

Figure 3. Ribosome residence times vs. $\alpha$. Plots of mean ribosome residence times on the entire mRNA lattice versus increasing translation initiation rates $(\alpha)$ [Top row:] Plots are for (a) D = 0.1 and (b) D = 1.0 at fixed values of correlation times, $\tau=0.1 \mathrm{~s}$ (orange), $\tau=1.0 \mathrm{~s}$ (green), $\tau=10 \mathrm{~s}$ (red). [Bottom row:] Same as top row for (c) $\tau=0.1 \mathrm{~s}$ and (d) $\tau=10.0 \mathrm{~s}$ for fixed values of diffusive strengths, $\mathrm{D}=$ 0.1 (orange), $\mathrm{D}=1.0$ (green) and $\mathrm{D}=2.0$ (red). All the plots are compared to that obtained with no EN in the translation initiation rate, $\alpha$ (blue). All the other parameters are fixed at $\beta=1.0 \mathrm{~s}^{-1}$ and $\gamma=1.0 \mathrm{~s}^{-1}$. 
In order to discern the effect of the two parameters of EN $(\tau$ and $D)$ on the translation process, we compare the ribosome residence times and the protein expression trends with those obtained for the no EN case. These are shown in Figs. 3 and 4. The simulations for these were carried out up to a maximum time of 8 minutes (which is the average lifetime of mRNAs in E. coli [62]) in each case. This allows for direct comparison of the number of proteins produced at the end of the simulation time. The curves show the mean and the standard error over mean (SEM) calculated from 100 such simulation runs.

These plots clearly show that for low diffusive strength ( $D=0.1$, Fig. 3a), the mean residence time increases slowly (non-negative slope greater than 1) with increasing $\alpha$. Further, with this low $D$, the curves are equivalent to those obtained in the no EN case even for higher $\tau$ values. The effect of EN manifests itself in the case of high $D(D=1.0$, Fig.3b), even when $\tau$ is low $(\tau=0.1 s)$. This is also reflected in Fig. 3c. However, as evident from Fig. 3(d), high $\tau$ combined with high $D$ leads to increased variation in the residence time.

We next compute the total number of proteins produced at the end of 8 minutes of real time in the simulation. For low $D$ (Fig. 4a), the number of proteins produced vs. $\alpha$ follows the same trend as the no EN case. However, for high $D$ (Figs. 4b, c and d), protein production increases with increasing $\alpha$ up to a point and then starts to flatten. This is a result of two opposing forces - (i) increase in translation rate due to increasing $\alpha$, which tends to raise protein production and (ii) an increase in the ribosome residence time (a consequence of increasing EN), which tends to decrease protein production. As evident from Figs. 4(b, c and d), the slope change (flattening) in these scenarios happens at successively lower $\alpha$ with increasing EN.

\section{Ribosome traffic in the presence of slow codons}

As mentioned earlier, many mRNAs invariably have certain codons or sequences of codons that slow down ribosome trafficking at that site. The effects of these slow codons are long lasting. We now explore their effects on mRNA translation, protein production and ribosome trafficking.
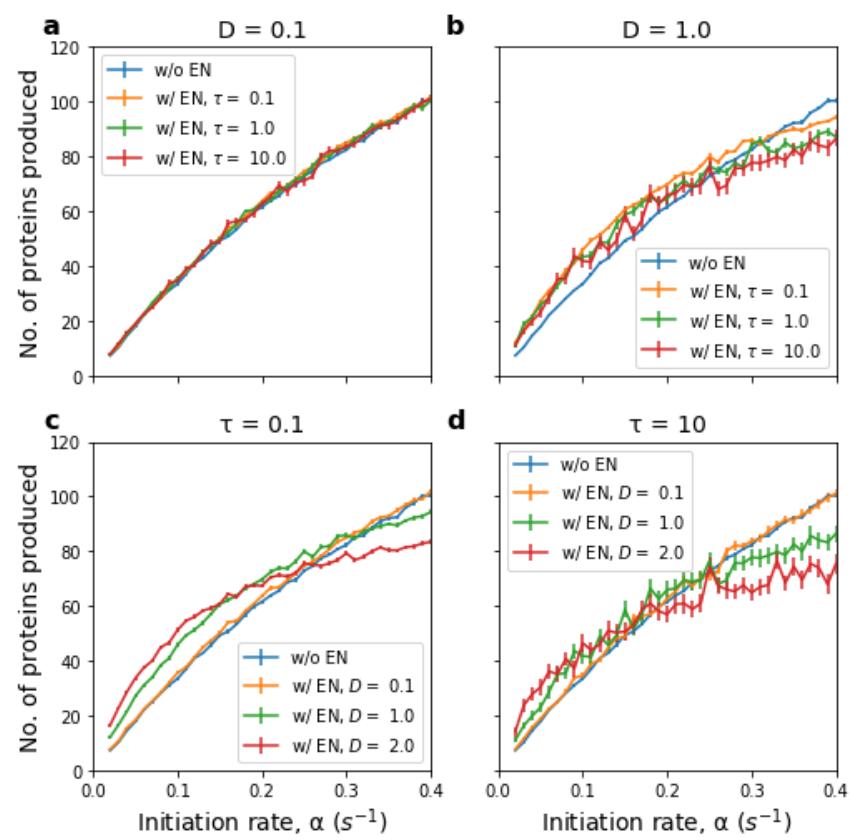

Figure 4. Protein expression vs. $\alpha$. Plots of mean number of proteins produced versus increasing translation initiation rate $(\alpha)$ [Top row:] Plots are for (a) D $=0.1$ and (b) D $=1.0$ at fixed values of correlation times, $\tau=0.1 \mathrm{~s}$ (orange), $\tau=1.0 \mathrm{~s}$ (green), $\tau=10 \mathrm{~s}$ (red). [Bottom row:] Same as top row for (c) $\tau=0.1 \mathrm{~s}$ and (d) $\tau=10.0 \mathrm{~s}$ for fixed values of diffusive strengths, $\mathrm{D}=0.1$ (orange), $\mathrm{D}=1.0$ (green) and $\mathrm{D}=2.0$ (red). All the plots are compared to that obtained with no $\mathrm{EN}$ in the translation initiation rate, $\alpha$ (blue). All the other parameters are fixed at $\beta=1.0 \mathrm{~s}^{-1}$ and $\gamma=1.0 \mathrm{~s}^{-1}$.

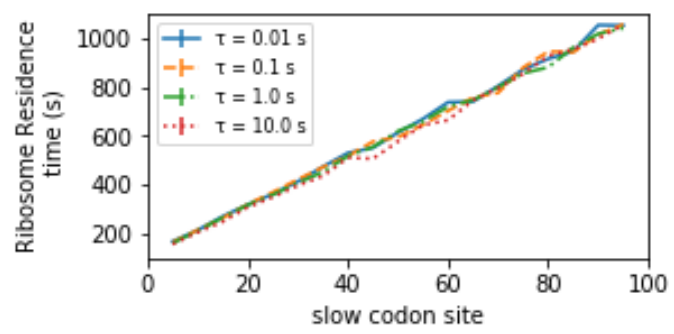

Figure 5. Effect of slow codons. Ribosome residence time versus the slow codon site (l) on the mRNA lattice for $\gamma=0.1 \mathrm{~s}^{-1}$ at the slow codon site, $\gamma=1.0 \mathrm{~s}^{-1}$ at all other sites, $\beta=1.0 \mathrm{~s}^{-1}, D=1.0$, and $\tau=0.01 \mathrm{~s}$ (blue solid line), $0.1 \mathrm{~s}$ (orange dashed line), $1.0 \mathrm{~s}$ (green dot dashed line) and $10 s$ (red dotted line).

We first look at the effect of the lattice site position of the slow codon on the mRNA. Referring to Fig. 1, the 
bioRxiv preprint doi: https://doi.org/10.1101/2020.08 21 260976; this version posted August 22, 2020. The copyright holder for this preprint (which was not certified by peer review) is the author/funder, who has granted bioRxiv a license to display the preprint in perpetuity. It is made available under aCC-BY-NC-ND 4.0 International license.
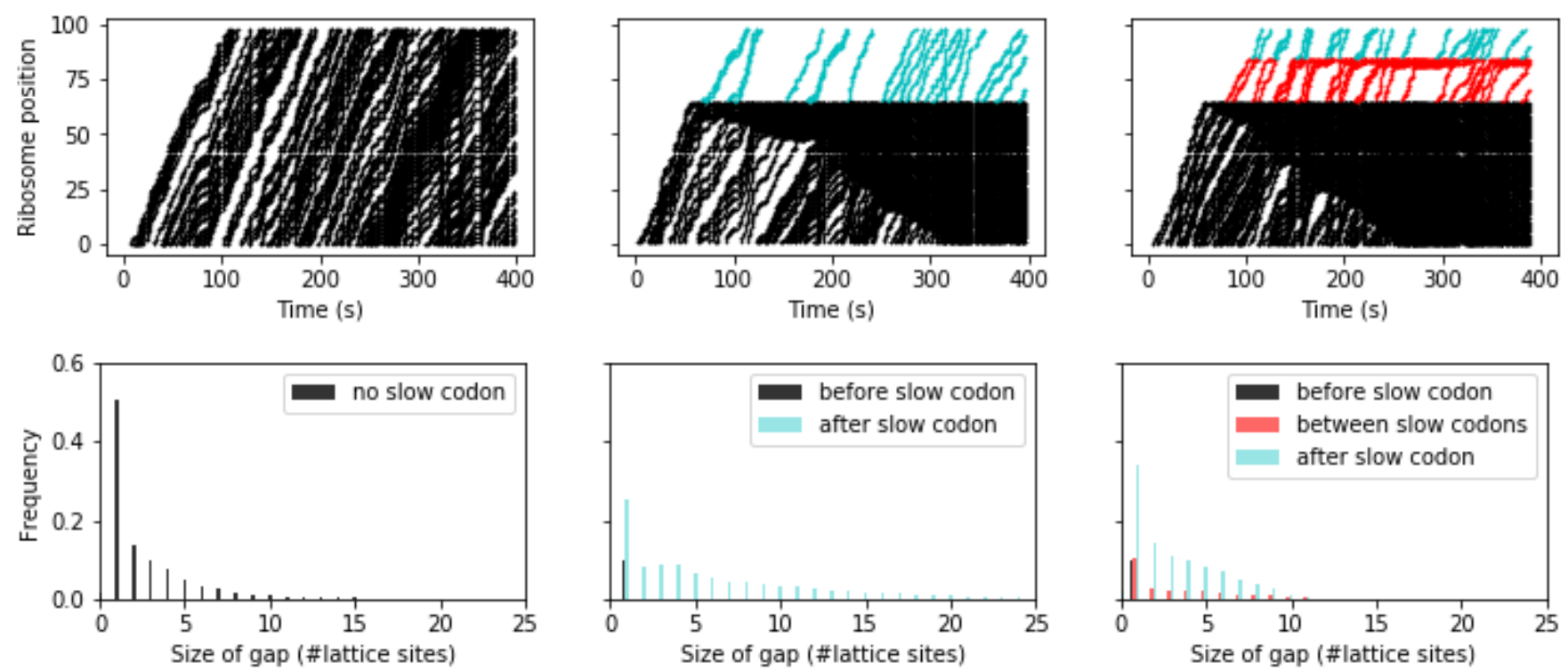

Figure 6. Ribosome trafficking. [Top:] Ribosomal position versus time and [Bottom:] frequency versus gap length between successive ribosomes on an mRNA with (a) no slow codons, (b) 1 slow codon at site $l=65$ and (c) two slow codons with the second slow codon site at $l=85$. Ribosomes and gaps before the first codon are shown in black, after the slow codons are shown in cyan and between two slow codons are shown in red. All the other parameters are fixed at $\alpha=0.4 s^{-1} \beta=1.0 s^{-1}$, $\gamma=0.1 \mathrm{~s}^{-1}$ for the slow codon site, $\gamma=1.0 \mathrm{~s}^{-1}$ for all other sites, $D=1.0$ and $\tau=1.0 \mathrm{~s}$. 
mRNA is considered to be composed of 100 lattice sites (l), with 1 representing the first site from the start codon and 100 the farthest on the right. With this picture, one can now see in Fig. 5, that the ribosome residence time increases linearly with the slow codon site. This can be a manifestation of the fact that the farther the slow codon site, the greater the traffic jam experienced by the ribosome on the mRNA lattice. Further, it seems that EN has no effect on the linear increase shown here when the elongation rate, $\gamma$, is decreased to 1 tenth the usual rate.

In the simulations represented by Fig. 5, we only considered one slow codon. We now also consider two slow codons placed 20 lattice sites apart and look at the ribosome trajectory and gaps. This is shown in Fig. 6. The slow codon sites are evident in the ribosome trajectories by the build up of ribosomes below that site. One can also observe that the build-up gets denser with two slow codons compared to just the one. This build-up is also reflected in the gap distribution among the three cases. mRNAs with no slow codons have a gap distribution that tapers off only at 15-20 lattice sites, whereas the positions before a slow codon have a gap no more than a single lattice site. The distribution of gaps broadens again beyond the slow codon, however it is more long-tailed than the no slow codon case. Finally, the trajectories beyond the two slow codons show lesser broadening in the gap distribution compared to the no slow codon case. Surprisingly, the no EN and with EN scenarios for the slow codon cases do not show any major difference between them. This could be because the slow codon masks the effect of EN.

\section{CONCLUSIONS}

$\mathbf{E N}$ in $\beta$. Since translation termination (kinetic rate $\beta$ ) is a rapid event compared to translation initiation (kinetic rate $\alpha$ ), as shown in Fig. 3, the order of magnitude of the noise that affects the rate $\alpha$ does nothing to perturb $\beta$. Of course, if the rate $\beta$ is made comparable to that of rate $\alpha=0.4 s^{-1}$, the ribosome residence time distributions show deviation from the gamma distribution. Not only that, in this scenario, the distribution obtained for the case of EN in beta is shifted to the left compared
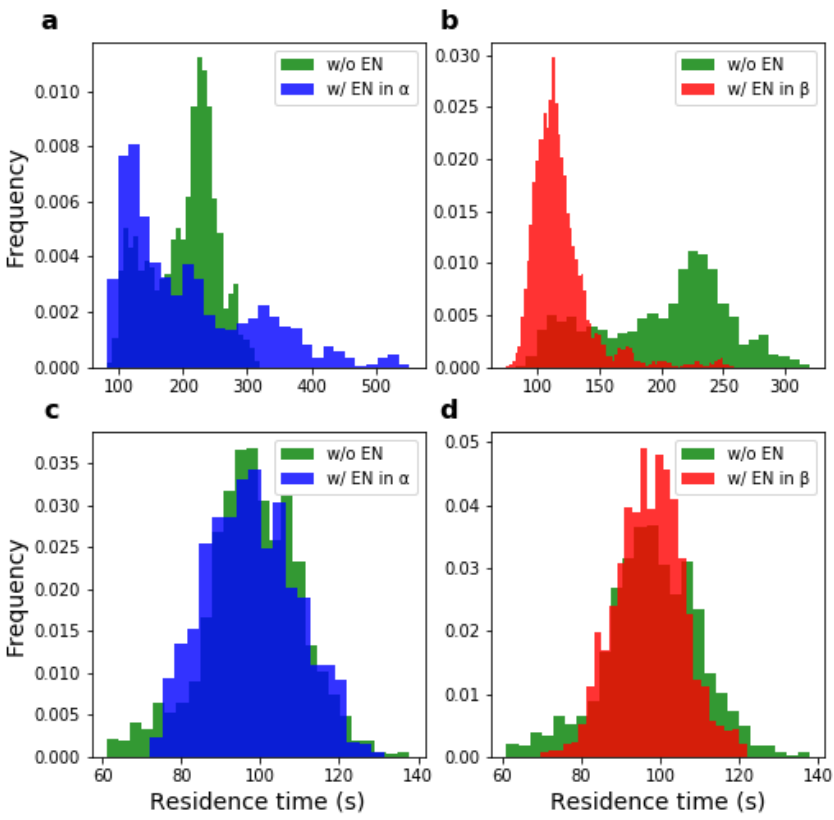

Figure 7. Effect of changing alpha to beta ratio on ribosome residence times. [Top:] Comparison of ribosome residence time distribution obtained from simulations with no EN and when EN is incorporated either in (a) the translation initiation rate $(\alpha)$ or $(\mathrm{b})$ the translation termination rate $(\beta)$ with $\langle\beta\rangle=0.4 s^{-1}$. [Bottom:] Same as top with $\langle\beta\rangle=$ $10.0 \mathrm{~s}^{-1}$. All the other parameters are fixed at $D=1.0, \tau=$ $1.0 s,\langle\alpha\rangle=0.4 s^{-1}$ and $\langle\gamma\rangle=1.0 s^{-1}$. Histograms are obtained from simulations. The overlaps in the distributions are shaded darker.

to the no EN case. Also, all the distributions are long tailed. This is shown in Figs. 7(a and b). However, when $\beta=10.0 s^{-1}>>\alpha$, as in Figs. $7(\mathrm{c}$ and $\mathrm{d})$, there is virtually no difference between the ribosome residence time distributions for the three cases, viz. no EN, EN in $\alpha$ and $\mathrm{EN}$ in $\beta$. The distributions in Figs. 7 (c and $\mathrm{d}$ ) also follow the gamma distributions. This analysis shows that the ratio between the three rates plays a major role in determining the nature of the residence time distributions. This then is also liable to affect protein production and gap distribution.

Protein production. In elucidating the effects of EN on mRNA translation, we see that the protein production shows a gradual increase with $\alpha$ (Fig. 4). This gradual increase also has two regimes, categorized by the slopes (high and low). For the low EN case (Fig. 4a), there is minimal change in the slope. However, for high EN (Fig. 
$4 \mathrm{~b}$, c and d), the change in slope is more pronounced. Further, a smooth transition is observed between the low and high slope regions of the curve. The property of gradual slope change is similar to the slope change observed in the ribosomal current in a previous study on mRNA translation in the yeast strain, S. cerevisiae [23]. Since protein production is a direct consequence of ribosomal current, the trend shown here is in agreement with expected behavior. However, the change in slope becoming more pronounced for high EN is a useful property that came out of this study. Since, number of proteins produced and its distribution is a quantity that can be determined from experiments, these slope changes can also be used to determine the extent of the extrinsic noise in the translation initiation rate.

Presence of slow codons. In our simulations, the slow codons act to mask the effect of $\mathrm{EN}$, i.e., they raise the mean ribosome residence time to such an extent that there is virtually no difference between the simulations with and without EN. Also, as indicated in Ciandrini et al's yeast analysis [23], the position of the slow codons does have an effect on the ribosomal current, which is attributed to a buildup of ribosomes before the slow codon site. This buildup manifests in the residence time distribution in our analysis. Here, we see in Fig. 5, that the buildup leads to a greater increase in the ribosome residence time even though there is no change in the rate. This buildup can also be seen in Fig. 6, which further changes the gap distribution profile.

Finally, this analysis of extrinsic noise in mRNA translation helps us understand and deduce the source of fluctuations in protein production. Specifically, under realistic conditions, noise in translation initiation has a major effect and through the analysis of protein production, one can even deduce the extent of that noise. Further, this analysis shows us that slow codons are also a source of fluctuation and the different gap distributions can be used to determine the presence of slow codons. One can also extend this study to include specific effects from diffusing ribosomes and drop off of these ribosomes from the mRNA lattice. These will be taken up in future studies. The analyses presented in this simulation study can also help in carrying out a similar analysis in experimental studies. This can then help in factoring in the effect of individual processes of mRNA translation that contribute to noisy protein distributions.
[1] Michael B Elowitz, Arnold J Levine, Eric D. Siggia, and Peter S Swain. Stochastic Gene Expression in a Single Cell. Science, 297(5584):1183-1186, 2002.

[2] Peter S Swain, Michael B Elowitz, and Eric D Siggia. Intrinsic and extrinsic contributions to stochasticity in gene expression. Proc. Nati. Acad. Sci. USA, 99(20):1279512800, 2002.

[3] Mukund Thattai and Alexander van Oudenaarden. Stochastic gene expression in fluctuating environments. Genetics, 167(1):523-30, may 2004.

[4] Marc S. Sherman, Kim Lorenz, M. Hunter Lanier, and Barak A. Cohen. Cell-to-Cell Variability in the Propensity to Transcribe Explains Correlated Fluctuations in Gene Expression. Cell Systems, 1(5):315-325, nov 2015.

[5] Philipp Thomas. Intrinsic and extrinsic noise of gene expression in lineage trees. Scientific Reports, 9:474, 2019.

[6] Rati Sharma and Elijah Roberts. Gradient sensing by a bistable regulatory motif enhances signal amplification but decreases accuracy in individual cells. Physical Biology, 13(3):036003, may 2016.

[7] Mahdi Golkaram, Stefan Hellander, Brian Drawert, and
Linda R. Petzold. Macromolecular Crowding Regulates the Gene Expression Profile by Limiting Diffusion. PLOS Computational Biology, 12(11):e1005122, nov 2016.

[8] Julien Mortier, Wubishet Tadesse, Sander K Govers, and Abram Aertsen. Stress-induced protein aggregates shape population heterogeneity in bacteria. Current Genetics, 65:865-869, 2019.

[9] Om Patange, Christian Schwall, Matt Jones, Casandra Villava, Douglas A Griffith, Andrew Phillips, and James C W Locke. Escherichia coli can survive stress by noisy growth modulation. Nature Communications, 9:5333, 2018.

[10] Aaron Vazquez-Jimenez and Jesus Rodriguez-Gonzalez. On Information Extraction and Decoding Mechanisms Improved by Noisy Amplification in Signaling Pathways. Scientific Reports, 9(1):1-14, 2019.

[11] Sangjin Kim and Christine Jacobs-Wagner. Effects of mRNA Degradation and Site-Specific Transcriptional Pausing on Protein Expression Noise. Biophysical Journal, 114(7):1718-1729, apr 2018.

[12] Oleg Yarchuk, Nathalie Jacques, Jean Guillerez, and 
Marc Dreyfus. Interdependence of translation, transcription and mrna degradation in the lacz gene. Journal of Molecular Biology, 226(3):581 - 596, 1992.

[13] Atillo Deanna and Joel G. Belasco. Lost in translation: the influence of ribosomes on bacterial mrna decay. Genes and Development, 19:2526-2533, 2005.

[14] Antoine Baudrimont, Vincent Jaquet, Sandrine Wallerich, Sylvia Voegeli, and Attila Becskei. Contribution of rna degradation to intrinsic and extrinsic noise in gene expression. Cell Reports, 26(13):3752 - 3761.e5, 2019.

[15] Tatsuya Morisaki and Timothy J. Stasevich. Quantifying single mRNA translation kinetics in living cells. Cold Spring Harbor Perspectives in Biology, 10(11), 2018.

[16] Bin Wu, Carolina Eliscovich, Young J. Yoon, and Robert H. Singer. Translation dynamics of single mRNAs in live cells and neurons. Science, 352(6292):1430-1435, 2016.

[17] Amin Espah Borujeni and Howard M. Salis. Translation Initiation is Controlled by RNA Folding Kinetics via a Ribosome Drafting Mechanism. Journal of the American Chemical Society, 138(22):7016-7023, 2016.

[18] Franka Voigt, Hui Zhang, Xianying A. Cui, Désirée Triebold, Ai Xin Liu, Jan Eglinger, Eliza S. Lee, Jeffrey A. Chao, and Alexander F. Palazzo. Single-Molecule Quantification of Translation-Dependent Association of mRNAs with the Endoplasmic Reticulum. Cell Reports, 21(13):3740-3753, 2017.

[19] Ivan L. Volkov, A. Carolin Seefeldt, and Magnus Johansson. Tracking of single tRNAs for translation kinetics measurements in chloramphenicol treated bacteria. Methods, 162-163(February):23-30, 2019.

[20] Deepak Khuperkar, Tim A. Hoek, Stijn Sonneveld, Bram M.P. Verhagen, Sanne Boersma, and Marvin E. Tanenbaum. Quantification of mRNA translation in live cells using single-molecule imaging. Nature Protocols, 15(April):1371-1398, 2020.

[21] Fuad Mohammad, Rachel Green, and Allen R. Buskirk. A systematically-revised ribosome profiling method for bacteria reveals pauses at single-codon resolution. eLife, $8: 1-25,2019$

[22] Shlomi Reuveni, Isaac Meilijson, Martin Kupiec, Eytan Ruppin, and Tamir Tuller. Genome-scale analysis of translation elongation with a ribosome flow model. PLoS Computational Biology, 7(9), 2011.

[23] Luca Ciandrini, Ian Stansfield, and M. Carmen Romano. Ribosome Traffic on mRNAs Maps to Gene Ontology: Genome-wide Quantification of Translation Initiation Rates and Polysome Size Regulation. PLoS Computational Biology, 9(1), 2013.
[24] Julien Racle, Adam Jan Stefaniuk, and Vassily Hatzimanikatis. Noise analysis of genome-scale protein synthesis using a discrete computational model of translation. The Journal of Chemical Physics, 143:44109, 2015.

[25] Pierre Bonnin, Norbert Kern, Neil T. Young, Ian Stansfield, and M. Carmen Romano. Novel mRNAspecific effects of ribosome drop-off on translation rate and polysome profile. PLoS Computational Biology, 13(5):e1005555, 2017.

[26] Elgin Korkmazhan, Hamid Teimouri, Neil Peterman, Erel Levine, and William Bialek. Dynamics of translation can determine the spatial organization of membranebound proteins and their mRNA. 114(51):13424-13429, 2017.

[27] Sandip Datta and Brian Seed. Influence of multiplicative stochastic variation on translational elongation rates. PLOS ONE, 13(1):e0191152, jan 2018.

[28] Heungwon Park and Arvind R. Subramaniam. Inverted translational control of eukaryotic gene expression by ribosome collisions. PLoS Biology, 17(9):e3000396, 2019.

[29] Itzik Nanikashvili, Yoram Zarai, Alexander Ovseevich, Tamir Tuller, and Michael Margaliot. Networks of ribosome flow models for modeling and analyzing intracellular traffic. Scientific Reports, 9(1):1-14, 2019.

[30] Stephan Uphoff. A Quantitative Model Explains SingleCell Dynamics of the Adaptive Response in Escherichia coli. Biophysical Journal, 117(6):1156-1165, sep 2019.

[31] Krešimir Josi, Manuel Ló Pez, William Ott, Liejune Shiau, Matthew $\mathrm{R}$ Bennett, and Jason M Haugh. Stochastic Delay Accelerates Signaling in Gene Networks. PLoS Comput Biol, 7(11), 2011.

[32] Srabanti Chaudhury. Modeling the effect of transcriptional noise on switching in gene networks in a genetic bistable switch. Journal of Biological Physics, 41(3):235246, jun 2015.

[33] Dao Guang Wang, Shaobing Wang, Bo Huang, and Feng Liu. Roles of cellular heterogeneity, intrinsic and extrinsic noise in variability of p53 oscillation. Scientific Reports, 9(1):5883, 2019.

[34] Damien Nicolas, Nick E. Phillips, and Felix Naef. What shapes eukaryotic transcriptional bursting? Mol. BioSyst., 13(7):1280-1290, jun 2017.

[35] Kuheli Biswas, Mayank Shreshtha, Anudeep Surendran, and Anandamohan Ghosh. First-passage time statistics of stochastic transcription process for time-dependent reaction rates. The European Physical Journal E, 42:24, 2019.

[36] Elijah Roberts, Shay Be'er, Chris Bohrer, Rati Sharma, and Michael Assaf. Dynamics of simple gene-network 
motifs subject to extrinsic fluctuations. Physical Review E, 92(6):062717, dec 2015.

[37] Marco Del Giudice, Stefano Bo, Silvia Grigolon, and Carla Bosia. On the role of extrinsic noise in microRNAmediated bimodal gene expression. PLoS Comput Biol, 14(4):e1006063, 2018.

[38] Michael Assaf, Elijah Roberts, Zaida Luthey-Schulten, and Nigel Goldenfeld. Extrinsic noise driven phenotype switching in a self-regulating gene. Physical Review Letters, 111(5):1-5, 2013.

[39] Niraj Kumar, Abhyudai Singh, and Rahul V. Kulkarni. Transcriptional Bursting in Gene Expression: Analytical Results for General Stochastic Models. PLOS Computational Biology, 11(10):e1004292, oct 2015.

[40] Sandip Kar, William T. Baumann, Mark R. Paul, and John J. Tyson. Exploring the roles of noise in the eukaryotic cell cycle. Proceedings of the National Academy of Sciences, 106(16):6471-6476, 2009.

[41] Tom Israeli and Michael Assaf. Population switching under a time-varying environment. Physical Review E, 101(2):022109, feb 2020.

[42] Peter Fekkes and Arnold J. M. Driessen. Protein Targeting to the Bacterial Cytoplasmic Membrane. Microbiology and Molecular Biology Reviews, 63(1):161-173, mar 1999.

[43] Jeffrey R. Moffitt, Shristi Pandey, Alistair N. Boettiger, Siyuan Wang, and Xiaowei Zhuang. Spatial organization shapes the turnover of a bacterial transcriptome. eLife, 5(MAY2016), may 2016.

[44] Keren Nevo-Dinur, Anat Nussbaum-Shochat, Sigal Ben-Yehuda, and Orna Amster-Choder. Translationindependent localization of mRNA in E. coli. Science, 331(6020):1081-1084, feb 2011.

[45] Avi Ad Avraam Buskilay, Shanmugapriya Kannaiahy, and Orna Amster-Choder. RNA localization in bacteria, aug 2014.

[46] Manuela Roggiani and Mark Goulian. ChromosomeMembrane Interactions in Bacteria. Annual Review of Genetics, 49(1):115-129, nov 2015.

[47] Anil K. Gorle, Amy L. Bottomley, Elizabeth J. Harry, J. Grant Collins, F. Richard Keene, and Clifford E. Woodward. DNA condensation in live E. coli provides evidence for transertion. Molecular BioSystems, 13(4):677680, mar 2017.

[48] B Derrida, M R Evanst, V Hakimf, and V Pasquiert. Exact solution of a ID asymmetric exclusion model using a matrix formulation. Technical report, 1993.

[49] Jan De Gier and Fabian H L Essler. Bethe ansatz solution of the asymmetric exclusion process with open bound- aries. Physical Review Letters, 95(24):1-4, 2005.

[50] Julien Cividini and Cécile Appert-Rolland. Zone clearance in an infinite TASEP with a step initial condition. Journal of Physics A: Mathematical and Theoretical, 50(26):265002, jun 2017.

[51] Milan Krbálek and Pavel Hrabák. Inter-particle gap distribution and spectral rigidity of the totally asymmetric simple exclusion process with open boundaries. J. Phys. A: Math. Theor, 44:175203-175225, 2011.

[52] Amin Espah Borujeni, Anirudh S. Channarasappa, and Howard M. Salis. Translation rate is controlled by coupled trade-offs between site accessibility, selective RNA unfolding and sliding at upstream standby sites. Nucleic Acids Research, 42(4):2646-2659, 22014.

[53] Guillaume Desnoyers, Marie Pier Bouchard, and Eric Massé. New insights into small RNA-dependent translational regulation in prokaryotes. Trends in Genetics, 29(2):92-98, feb 2013.

[54] Christina E. Brule and Elizabeth J. Grayhack. Synonymous codons: Choose wisely for expression. Trends in Genetics, 33(4):283 - 297, 2017.

[55] Anand Pai and Lingchong You. Optimal tuning of bacterial sensing potential. Molecular Systems Biology, 5(1):286, 2009.

[56] Leah B. Shaw, R. K. P. Zia, and Kelvin H. Lee. Totally asymmetric exclusion process with extended objects: A model for protein synthesis. Phys. Rev. E, 68:021910, Aug 2003.

[57] Mary J Dunlop, Robert Cox, Joseph H Levine, Richard M Murray, and Michael B Elowitz. Regulatory activity revealed by dynamic correlations in gene expression noise. Nature Genetics, 40:1493-1498, December 2008.

[58] Michał Komorowski, Bärbel Finkenstädt, and David Rand. Using a single fluorescent reporter gene to infer half-life of extrinsic noise and other parameters of gene expression. Biophysical Journal, 98(12):2759 - 2769, 2010.

[59] Emma M. Keizer, Björn Bastian, Robert W. Smith, Ramon Grima, and Christian Fleck. Extending the linearnoise approximation to biochemical systems influenced by intrinsic noise and slow lognormally distributed extrinsic noise. Phys. Rev. E, 99:052417, May 2019.

[60] Daniel T Gillespie. Exact Stochastic Simulation of Coupled Chemical Reactions. Journal of Physical Chemistry, 81:2340-2361, 1977.

[61] M Voliotis, P Thomas, R Grima, and C G Bowsher. Stochastic Simulation of Biomolecular Networks in Dynamic Environments. PLOS Computational Biology, 
12(6):1004923, 2016.

[62] Jonathan A. Bernstein, Arkady B. Khodursky, Pei-Hsun Lin, Sue Lin-Chao, and Stanley N. Cohen. Global anal- ysis of mrna decay and abundance in escherichia coli at single-gene resolution using two-color fluorescent dna microarrays. Proceedings of the National Academy of Sciences, 99(15):9697-9702, 2002. 